\title{
Uma Proposta de Oficina de Desenvolvimento de Jogos Digitais para Ensino de Programação
}

\author{
José Raul B. Andrade, Jonas M. Targino, Wesley K. Francisco de Oliveira, \\ Ana Liz Souto Oliveira de Araújo, Flávia Veloso Costa Souza \\ Departamento de Ciências Exatas - Universidade Federal da Paraíba \\ Rua da Mangueira, s/n, Companhia de Tecidos Rio Tinto \\ 58.297-000 - Rio Tinto - PB - Brasil \\ \{raul.andrade, jonas.mendonca, wesley.kelvyn, analiz,flavia\}@dcx.ufpb.br
}

\begin{abstract}
Game development stimulates multidisciplinary skills, such as programming, teamwork and creativity. This paper describes an approach to stimulate game programming and multidisciplinary skills in high school. We use Scratch and encourage competition among groups. The results show that students documented the process, developed cognitive skills related to programming and used heuristics in their games.
\end{abstract}

Resumo. O processo de desenvolvimento de um jogo digital trabalha habilidades multidisciplinares, como programação, trabalho em equipe, criticidade e criatividade. O presente trabalho descreve uma abordagem para estimular programação no ensino médio por meio do desenvolvimento de jogos digitais, dando suporte à multidisciplinaridade intrínseca ao processo. Utilizamos a ferramenta Scratch e estimulamos a competitividade entre os grupos. Os resultados apontaram que os alunos conseguiram documentar o processo de produção, desenvolveram habilidades cognitivas relacionadas à programação e empregaram heurísticas de jogabilidade nos jogos produzidos.

\section{Introdução}

Nos últimos anos, em diversos países, o número de alunos que ingressam em cursos de Computação e Tecnologia da Informação (TI) vem diminuindo [Vegso, 2010]. Na França, por exemplo, entre 2005 e 2009, o número de alunos de Ciência da Computação caiu 25\% [Muratet et al., 2009]. Além disso, durante a década dos anos 2000, foi registrada uma diminuição de 50\% do número de alunos americanos que tem interesse nos cursos dessa área [Crenshaw et al., 2008]. No contexto nacional a situação não é diferente. Um estudo realizado por Hernandez et al. (2010), comparando uma universidade brasileira com uma americana, aponta que a diminuição do interesse dos estudantes pela área em questão é equivalente nos dois países.

Um dos fatores que podem interferir no decremento do número de egressos nos cursos de TI é a maneira como conceitos e práticas da computação são apresentados, ou nem são apresentados, para os alunos na educação básica [Barcelos e Silveira, 2012]. Esses estudantes deveriam ser introduzidos aos conceitos da Ciência da Computação de 
V Congresso Brasileiro de Informática na Educação (CBIE 2016)

Anais dos Workshops do V Congresso Brasileiro de Informática na Educação (CBIE 2016)

forma prática e motivadora, fazendo-os entender de fato a sua importância e aplicabilidade em seu cotidiano.

De acordo com Medeiros et al. (2013), utilizar jogos em práticas pedagógicas podem trazer grandes benefícios no processo de ensino e aprendizagem. Segundo Moratori (2003), os jogos são capazes de trabalhar habilidades mentais, físicas e didáticas de maneira simultânea. Assim como a utilização dos jogos, o processo de desenvolvimento deles apresenta benefícios. Esse processo pode estimular a autonomia, a criatividade, a criticidade e o trabalho em equipe, além de ser uma forma estimulante de abordar os conteúdos de programação.

São encontradas na literatura algumas propostas para ensinar a desenvolver jogos. Porém, dentre elas são identificados diferentes objetivos para a utilização dessa prática. Andrade (2013) propõe a criação de jogos com intuito de abordar conteúdos de matemática no ensino médio. Ainda com esse público alvo, Rebouças et al (2010) propõem formas de aumentar o interesse dos alunos pela área de informática e Scaico et al (2012) propõe desenvolver jogos para trabalhar o ensino de programação, assim como estimular o Pensamento Computacional.

Neste contexto, planejamos, executamos e avaliamos a Oficina de Produção de Jogos. O objetivo principal foi abordar os aspectos multidisciplinares intrínsecos ao processo de desenvolvimento de jogos para estimular o ensino de programação no Ensino Médio. A oficina abordou o processo de concepção da ideia do jogo (produção de documentos e uso de técnicas auxiliares), a menção dos conteúdos introdutórios de marketing e publicidade em jogos e a utilização de heurísticas de jogabilidade ainda na etapa de elaboração do jogo. Essas heurísticas são um conjunto de características que devem ser consideradas em um jogo, relacionadas à interação do usuário com a aplicação. Além dos conteúdos abordados, também foram adotados o trabalho em equipe e o estímulo à competitividade como fatores motivacionais.

O trabalho está organizado da seguinte maneira: a seção 2 cita trabalhos relacionados; a seção 3 explica o processo de planejamento da oficina; a seção 4 expõe os resultados no contexto de desenvolvimento de jogos; e por último a seção 5 apresenta as considerações finais e trabalhos futuros.

\section{Trabalhos Relacionados}

$\mathrm{Na}$ literatura são encontradas diferentes propostas nas quais o desenvolvimento de jogos é abordado para finalidades pedagógicas. O trabalho de Andrade et al. (2013) teve como objetivo promover o ensino da matemática por meio do desenvolvimento de jogos usando o Scratch. No mesmo contexto, Barcelos e Silveira (2013) realizaram atividades práticas de desenvolvimento de jogos digitas e investigaram habilidades do Pensamento Computacional relacionadas à matemática.

França e Tedesco (2015) promoveram o desenvolvimento de jogos em duas turmas do ensino médio usando a ferramenta Stencyl (http://www.stencyl.com/). O trabalho analisou o processo de avaliação por pares e a qualidade do jogos digitais produzidos pelos estudantes. Os resultados demonstraram melhoras na qualidade no aspecto de jogabilidade dos jogos criados nas duas turmas, em especial na turma experimental (com avaliação por pares). 
V Congresso Brasileiro de Informática na Educação (CBIE 2016)

Anais dos Workshops do V Congresso Brasileiro de Informática na Educação (CBIE 2016)

\section{Elaboração da Oficina de Produção de Jogos}

A proposta de intervenção pedagógica apresentada e avaliada neste trabalho é resultado de pesquisa bibliográfica e de duas oficinas pilotos. Em cada oficina piloto, aplicamos um questionário aos participantes com o objetivo de identificar os aspectos positivos e negativos da mesma. Ademais, também discutimos a avaliação da oficina e registramos o processo de aperfeiçoamento da proposta. A percepção dos envolvidos foi coletada e esquematizada nesta seção.

\subsection{Planejamento da Oficina}

Além da familiaridade do público-alvo com jogos eletrônicos, a escolha dessa abordagem se deu pela abrangência de habilidades que o desenvolvimento de jogos estimula, como: autonomia, criatividade, criticidade e trabalho em equipe. Desde a idealização, a Oficina de Produção de Jogos buscou dar suporte às características multidisciplinares intrínsecas ao processo de produção de um jogo.

Para a seleção dos temas a serem abordados, foi considerado os conteúdos de programação (variáveis, operadores lógicos e as estruturas sequenciais, condicionais e de repetição) e aspectos criativos (criação e edição de imagens, efeitos sonoros e concepção da narrativa). Scratch (https://scratch.mit.edu/) foi a plataforma escolhida para os alunos desenvolverem os jogos. A sua escolha como ambiente de desenvolvimento foi motivada pela facilidade de uso, legibilidade dos códigos, variedade de recursos disponibilizados e versão off-line da ferramenta.

A estrutura básica da oficina foi composta por encontros presenciais, com duração de 3 horas cada. Ao final de cada oficina piloto, os alunos desenvolveram um jogo (projeto final) praticando o conteúdo abordado no decorrer da oficina. Para estimular a participação e o aprendizado dos alunos, adicionamos como fator motivacional a competitividade: o melhor jogo seria premiado no final. A seguir são apresentadas as adaptações realizadas a partir das experiências aprendidas nas oficinas piloto até chegar nesta proposta de intervenção.

\subsubsection{Primeira Oficina Piloto}

A primeira oficina piloto aconteceu no Laboratório de Informática da UFPB campus IV em Rio Tinto. Participaram 6 alunos do $1^{\circ}$ ano do Ensino Médio de uma escola estadual da região, conveniada ao PIBID (Programa Institucional de Bolsa de Iniciação à Docência) de Computação. Essa oficina piloto foi composta por 2 encontros de 3 horas cada. Trabalhamos os conteúdos de programação (variáveis, operadores lógicos e estrutura de repetição) com exemplos de jogos já implementados no Scratch. Nesta etapa, a criatividade foi explorada apenas por meio da personalização, ou seja, os alunos desenvolveram jogos com a mecânica semelhante aos exemplos apresentados, porém caracterizando as imagens e sons utilizados. Além disso, apesar de incentivar a discussão de ideias entre os alunos, a produção dos projetos finais foi individual.

A partir do feedback dos alunos e discussão interna da equipe, identificamos as seguintes limitações: (i) o número de encontros foi insuficiente para tratar dos conteúdos propostos; (ii) os alunos se motivavam com a prática da programação, porém ficavam confusos com a nomenclatura dos conceitos; e (iii) devido à ausência de 
V Congresso Brasileiro de Informática na Educação (CBIE 2016)

Anais dos Workshops do V Congresso Brasileiro de Informática na Educação (CBIE 2016)

documentação, aspectos da concepção da ideia do jogo eram criados ou alterados na etapa de programação. Isso dificultou o gerenciamento do tempo para conclusão do jogo e refletiu na qualidade do resultado final.

\subsubsection{Segunda e Terceira Oficinas Piloto}

A segunda e a terceira oficinas piloto ocorreram de forma semelhante. Ambas aconteceram no Laboratório de Informática de uma escola pública estadual no município de Mamanguape, Paraíba, conveniada do projeto PIBID de Computação. Em cada uma das edições participaram 13 alunos do $1^{\circ}$ e $2^{\circ}$ ano do Ensino Médio.

Com intuito de sanar as dificuldades identificadas da primeira oficina piloto, realizamos modificações no conteúdo e organização. As alterações foram as seguintes: (i) três encontros de três horas cada; (ii) o conteúdo de programação passou a ser abordado de forma prática; e (iii) a documentação do jogo (storyboards e diagrama de elementos do jogo) foi incluída nos conteúdos trabalhados na oficina. A Figura 1 descreve o conteúdo básico do modelo do diagrama de elementos de jogo apresentado aos alunos. Ademais, a produção passou a ser em duplas e, diferente da edição anterior, na qual os alunos adaptavam o código dos jogos apresentados pelos instrutores, a mecânica e o código dos jogos foram definidos e implementados pelos alunos.

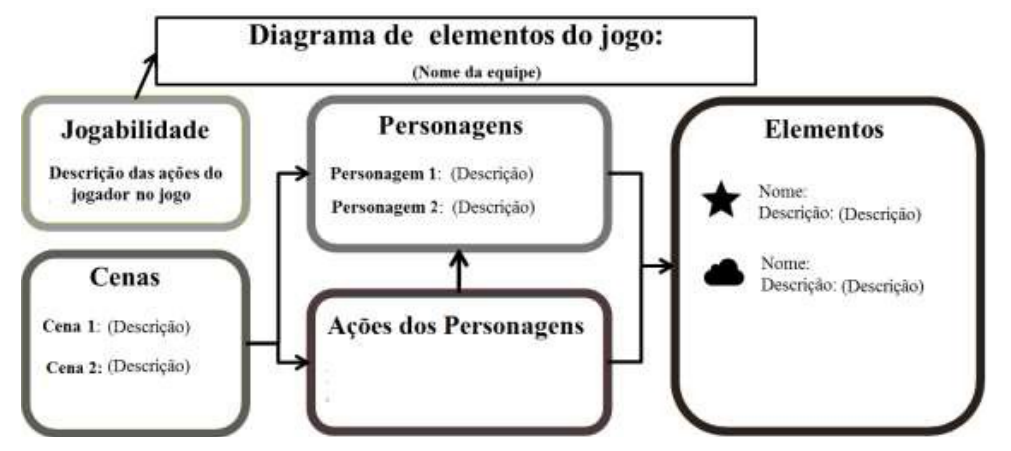

Figura 1. Modelo do diagrama de elementos do jogo

No conteúdo da oficina, acrescentamos também conceitos de heurísticas de jogabilidade. Essas heurísticas abrangem um conjunto de características relacionadas à usabilidade nos jogos, influenciando diretamente no processo de elaboração de sua mecânica. Como resultado dessas alterações, os jogos foram melhor elaborados e o processo de desenvolvimento mais organizado. As duplas se auto-organizaram na divisão das tarefas de acordo com a habilidade de cada membro. Apesar das melhorias listadas, notamos que a quantidade de encontros ainda não era o suficiente para desenvolver todo o conteúdo.

\subsection{Oficina de Produção de Jogos}

A Oficina de Produção de Jogos atual é composta por 5 aulas presenciais de 3 horas cada. Assim, continuamos os conteúdos de heurísticas de jogabilidade, devido aos resultados positivos de sua aplicação no piloto, como também a etapa de documentação (storyboard e diagrama de elementos básicos do jogo). Nesta edição, incluímos os assuntos de marketing e publicidade em jogos, bem como as possibilidades de aplicação do jogo, como: educação, saúde e treinamento. Contextualizamos esses assuntos no 
V Congresso Brasileiro de Informática na Educação (CBIE 2016)

Anais dos Workshops do V Congresso Brasileiro de Informática na Educação (CBIE 2016)

mercado atual de jogo no estado da Paraíba.

Nesta edição, a competitividade entre equipes foi mais estimulada. Ocorreu a minimaratona de programação e o desafio de publicidade dos jogos desenvolvidos nessa minimaratona. Além dessas duas competições, a documentação (storyboard e diagrama de elementos do jogo) e o jogo desenvolvido no final do curso somam uma pontuação. A dupla que mais pontuou recebeu a medalha de campeã.

O Quadro 1 apresenta as informações gerais sobre a organização dos conteúdos por aula. Na aula 1 introduzimos conceitos de jogos e o ambiente Scratch. Na aula 2 trabalhamos conceitos e práticas de programação. Na aula 3 apresentamos heurísticas de jogabilidade e realizamos a minimaratona de programação. $\mathrm{Na}$ aula 4 trabalhamos a concepção do projeto. Por último, na aula 5, os alunos desenvolveram seus projetos. Informações detalhadas são apresentadas no site da oficina: http://bit.ly/oficinadejogos

Quadro 1. Organização de conteúdos e práticas por aula.

\begin{tabular}{|l|l|}
\hline \multicolumn{1}{|c|}{ Aulas } & \multicolumn{1}{c|}{ Conteúdos e atividades } \\
\hline Aula 01: Mercado de jogos & $\begin{array}{l}\text { Breve história dos jogos. Mercado de jogos. } \\
\text { Introdução ao ambiente Scratch e à programação. }\end{array}$ \\
\hline Aula 02: Programação para jogos & Desenvolvimento de jogos e programação. \\
\hline Aula 03: Mecânicas do jogo & Heurísticas de jogabilidade. Minimaratona de programação. \\
\hline Aula 04: Concepção do projeto do jogo & $\begin{array}{l}\text { Marketing em games. Aplicações de jogos. } \\
\text { Diagrama de elementos do jogo e storyboard. }\end{array}$ \\
\hline Aula 05: Prática & Desenvolvimento do projeto. \\
\hline
\end{tabular}

\section{Resultados e Discussões}

A Figura 2 mostra as telas dos projetos finais produzidos pelos alunos. Analisamos e discutimos esses projetos sob três aspectos: documentação (etapa de planejamento), programação (ensino do conteúdo de programação) e jogabilidade (heurísticas do jogo).

Os participantes desta última Oficina de Produção de Jogos foram 13 alunos do $1^{\circ}$ e $2^{\circ}$ ano do Ensino Médio da escola pública em Mamanguape/PB conveniada ao PIBID. A média de idade da turma foi de 16 anos. Os alunos não tinham conhecimentos prévios em programação. Todos usavam a internet com frequência diária para fins de estudo e/ou lazer e todos afirmaram gostar de jogos eletrônicos.

\subsection{Documentação (diagrama e storyboard)}

O diagrama de elementos do jogo proporcionou as duplas pensarem de forma mais ampla no que é necessário conter no jogo, evitando assim, criar novas funcionalidades na etapa de programação (atitude que tinha sido observada nas oficinas piloto). Quanto aos storyboards, os alunos mostraram-se empolgados, principalmente por ser uma atividade lúdica, na qual eles criavam os personagens, história e diagramas de sequência do jogo. Essas atividades foram realizadas manualmente pelos alunos no papel ou na cartolina, conforme mostra a Figura 3. Nessa etapa, observamos que os estudantes com dificuldade em programação demonstraram habilidade no desenho, na criação da narrativa e na organização do projeto. Mesmo com essa observação, não houve divisão 
V Congresso Brasileiro de Informática na Educação (CBIE 2016)

Anais dos Workshops do V Congresso Brasileiro de Informática na Educação (CBIE 2016)

fixa de atividade na dupla, ou seja, ambos trabalharam em todas as etapas do processo.
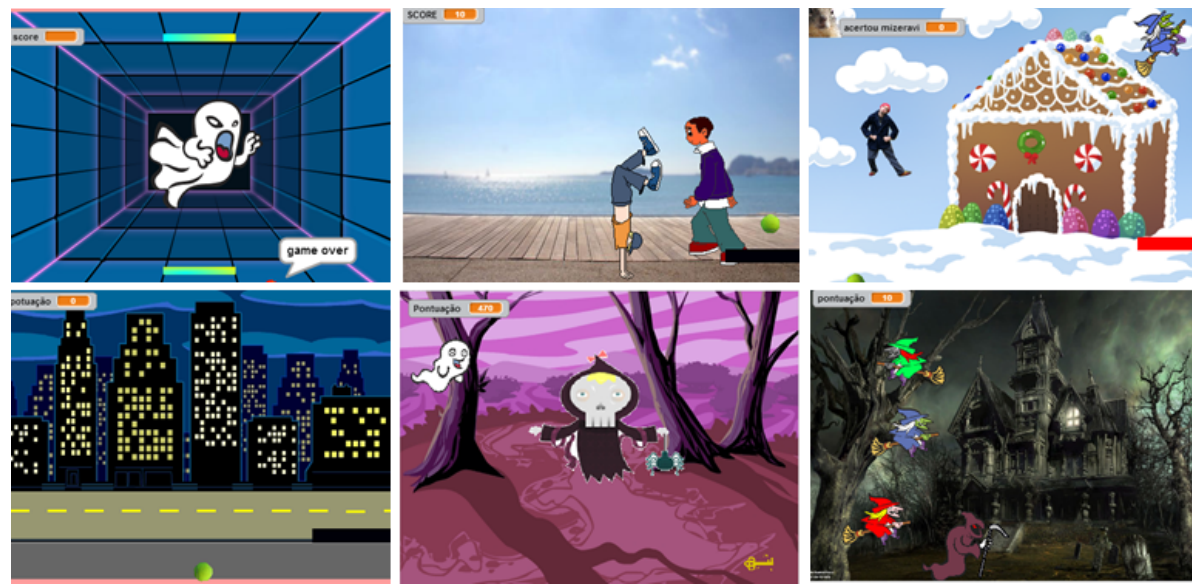

Figura 2. Tela dos jogos desenvolvidos pelos alunos durante a oficina

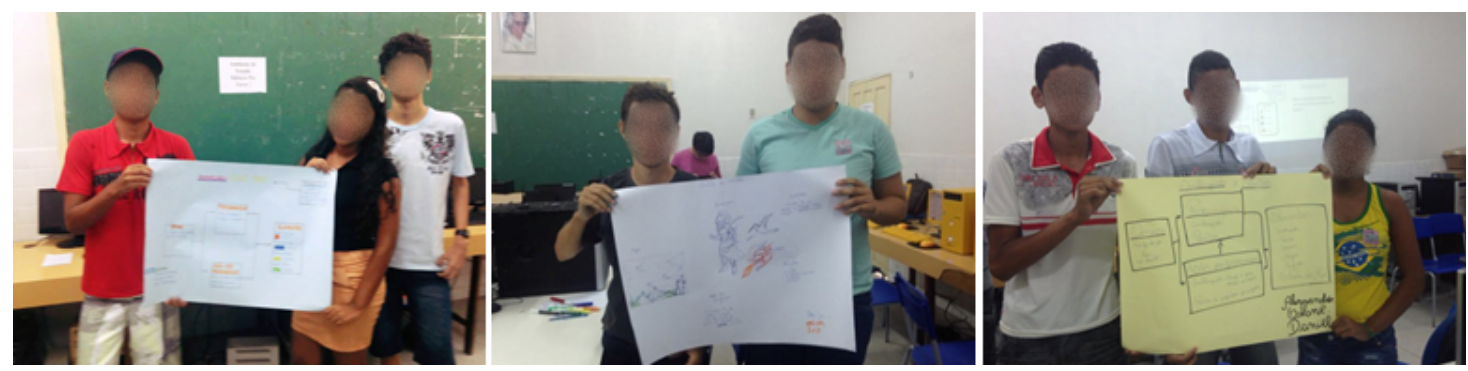

Figura 3. Documentação produzida pelos alunos durante a oficina

\subsection{Programação}

Para avaliar o ensino de conteúdos de programação, os projetos finais foram avaliados utilizando a ferramenta Dr. Scratch ${ }^{1}$. Dr. Scratch é uma ferramenta web gratuita para análise automática de projetos Scratch. A versão atual fornece informações sobre o projeto em termos de lógica, paralelismo, interatividade, representação de dados, controle de fluxo, sincronização e abstração. Informações mais detalhadas dos critérios de avaliação são apresentadas no Quadro 2.

Os critérios apresentados no Quadro 2 são avaliados em uma escala de zero a três, onde zero significa que o projeto não abordou o critério e três significa que abordou da forma mais completa. Organizamos os projetos dos alunos nomeando-os de A a F, submetemos os códigos ao Dr. Scratch e obtivemos os resultados apresentados no Quadro 3.

De acordo com os dados sumarizados no Quadro 3, os critérios de lógica e abstração são os mais ausentes nos projetos. Esses critérios estão relacionados à qualidade e reutilização do código. $\mathrm{O}$ aspecto lógico está ligado à possibilidade de adaptação de um trecho de código para diferentes ações. Já o critério de abstração verifica a decomposição de um problema em problemas menores, para facilitar o entendimento, a programação e a depuração. Assim, identificamos que os alunos

1 Disponível em: http://drscratch.programamos.es/ 
V Congresso Brasileiro de Informática na Educação (CBIE 2016)

Anais dos Workshops do V Congresso Brasileiro de Informática na Educação (CBIE 2016)

tiveram pouca preocupação na qualidade do código e mais foco na execução correta. Isso pode ser resultado da forma prática como a programação foi ensinada em detrimento de orientações teóricas, pois não abordamos, de forma direta, boas práticas de organização e reutilização de código.

Quadro 2. Critérios de avaliação adotados no Dr Scratch.

\begin{tabular}{|c|l|}
\hline Critério & \multicolumn{1}{c|}{ O que é avaliado? } \\
\hline Lógica & $\begin{array}{l}\text { Instruções lógicas do código e dinamicidade (diferentes ações ocorrem } \\
\text { dependendo do contexto). }\end{array}$ \\
\hline Paralelismo & A possibilidade dos objetos do jogo executarem ações simultaneamente. \\
\hline Interatividade & $\begin{array}{l}\text { A possibilidade de outros usuários conseguirem criar novas funcionalidades } \\
\text { para o jogo desenvolvido. }\end{array}$ \\
\hline $\begin{array}{c}\text { Representação de } \\
\text { dados }\end{array}$ & $\begin{array}{l}\text { O conjunto de informações sobre os objetos do jogo que permitem seu } \\
\text { funcionamento correto. }\end{array}$ \\
\hline $\begin{array}{c}\text { Controle de fluxo } \\
\text { Sincronização }\end{array}$ & O controle (direção, posição e movimentação) dos objetos do jogo. \\
\hline Abstração & A divisão de um problema em partes menores para facilitar a resolução. \\
\hline
\end{tabular}

A iteratividade, representação de dados, controle de fluxo e sincronização estiveram presentes nos jogos (pontuação $2 / 3$ na maioria dos projetos). $O$ paralelismo foi o critério mais presente nos projetos. Acredita-se que isso ocorreu uma vez que a utilização incorreta desse critério afeta diretamente na execução do jogo. Isso torna esse aspecto mais visível e, consequentemente, mais urgente de conserto. Como é possível identificar no Quadro 3, o Projeto A possui o melhor resultado e, somando as outras etapas de avaliação, foi o projeto vencedor da oficina.

Quadro 3. Resultado de avaliação dos projetos fornecidos pelo Dr Scratch.

\begin{tabular}{|c|c|c|c|c|c|c|}
\hline Critérios & Projeto A & Projeto B & Projeto C & Projeto D & Projeto E & Projeto F \\
\hline Lógica & $3 / 3$ & $1 / 3$ & $1 / 3$ & $1 / 3$ & $1 / 3$ & $3 / 3$ \\
\hline Paralelismo & $3 / 3$ & $1 / 3$ & $3 / 3$ & $2 / 3$ & $3 / 3$ & $1 / 3$ \\
\hline Interatividade & $2 / 3$ & $2 / 3$ & $2 / 3$ & $2 / 3$ & $2 / 3$ & $2 / 3$ \\
\hline Representação de dados & $2 / 3$ & $2 / 3$ & $1 / 3$ & $2 / 3$ & $2 / 3$ & $2 / 3$ \\
\hline Controle de fluxo & $2 / 3$ & $2 / 3$ & $2 / 3$ & $2 / 3$ & $2 / 3$ & $2 / 3$ \\
\hline Sincronização & $2 / 3$ & $2 / 3$ & $2 / 3$ & $2 / 3$ & $2 / 3$ & $2 / 3$ \\
\hline Abstração & $1 / 3$ & $1 / 3$ & $1 / 3$ & $1 / 3$ & $1 / 3$ & $1 / 3$ \\
\hline
\end{tabular}

\subsection{Jogabilidade}

Para avaliar a jogabilidade dos projetos, utilizamos as heurísticas propostas por Korhonen e Koivisto [2006] apresentadas no Quadro 4. A objetividade e clareza dos autores motivaram a escolha. Os projetos foram avaliados individualmente pelos membros da equipe e foi realizada uma votação majoritária para determinar a presença ou ausência de cada heurística. O Quadro 5 apresenta o resultado da avaliação heurística dos projetos desenvolvidos na oficina. Em relação à clareza de informações para o usuário (H3, H4, H5, H6 e H8), a maior parte dos jogos apresentou instruções claras e 
V Congresso Brasileiro de Informática na Educação (CBIE 2016)

Anais dos Workshops do V Congresso Brasileiro de Informática na Educação (CBIE 2016)

intuitivas e a disposição dos elementos seguiam padrões comuns (apenas o projeto $\mathrm{E}$ não apresentou a heurística H5 e o projeto D não apresentou a heurística H8).

Quadro 4. Heurísticas, segundo Korhonen e Koivisto [2006].

\begin{tabular}{|c|l|}
\hline Heurística & \multicolumn{1}{|c|}{ Jogabilidade } \\
\hline H1 & Não gaste o tempo do jogador \\
\hline H2 & Prepare-se para as interrupções \\
\hline H3 & Leve em conta outras pessoas \\
\hline H4 & Siga convenções padrão \\
\hline H5 & Fornecer ajuda gameplay \\
\hline H6 & Use termos que são familiares ao jogador \\
\hline H7 & O estado dos personagens e o jogo devem ser claramente visíveis \\
\hline H8 & O jogador deve ter objetivos claros \\
\hline H9 & Apoiar diversos jogadores e estilos de jogo \\
\hline H10 & Não incentivar tarefas repetitivas e aborrecidas \\
\hline
\end{tabular}

A organização e a otimização da jogabilidade (H1, H3 e H10) também foram empregadas nos projetos (apenas o projeto A não apresentou $\mathrm{H} 1$ e os projetos $\mathrm{B}$ e $\mathrm{C}$, a H10). Vimos que os alunos tiveram a preocupação de serem objetivos, evitando a perda de tempo do jogador e tarefas cansativas. As heurísticas relacionadas às interrupções durante a execução do jogo $(\mathrm{H} 2)$, clareza do estado emocional dos personagens $(\mathrm{H} 7)$ e apoio a diferentes estilos de jogo (H9) não puderam ser adotadas devido à ferramenta Scratch apresentar limitações quanto ao gráfico e a comunicação com outras aplicações.

Quadro 5. Avaliação de heurística de jogabilidade nos projetos dos alunos

\begin{tabular}{|c|c|c|c|c|c|c|c|c|c|c|}
\hline Projetos & H1 & H2 & H3 & H4 & H5 & H6 & H7 & H8 & H9 & H10 \\
\hline Projeto A & & & $\mathrm{X}$ & $\mathrm{X}$ & $\mathrm{X}$ & $\mathrm{X}$ & & $\mathrm{X}$ & & $\mathrm{X}$ \\
\hline Projeto B & $\mathrm{X}$ & & $\mathrm{X}$ & $\mathrm{X}$ & $\mathrm{X}$ & $\mathrm{X}$ & & $\mathrm{X}$ & & \\
\hline Projeto C & $\mathrm{X}$ & & $\mathrm{X}$ & $\mathrm{X}$ & $\mathrm{X}$ & $\mathrm{X}$ & & $\mathrm{X}$ & & \\
\hline Projeto D & $\mathrm{X}$ & & $\mathrm{X}$ & $\mathrm{X}$ & $\mathrm{X}$ & $\mathrm{X}$ & & & & $\mathrm{X}$ \\
\hline Projeto E & $\mathrm{X}$ & & $\mathrm{X}$ & $\mathrm{X}$ & & $\mathrm{X}$ & & $\mathrm{X}$ & & $\mathrm{X}$ \\
\hline Projeto F & $\mathrm{X}$ & & $\mathrm{X}$ & $\mathrm{X}$ & $\mathrm{X}$ & $\mathrm{X}$ & & $\mathrm{X}$ & & $\mathrm{X}$ \\
\hline
\end{tabular}

\subsection{Análise Realizada pelos Alunos}

Em um questionário aplicado ao final da oficina, investigamos o ponto de vista dos alunos em relação a oficina e sugestões de melhorias. Perguntamos aos alunos qual a opinião deles a respeito da oficina e quais as sugestões de melhorias para as próximas oficinas. Segundo os alunos, o curso ajudou a compreender a mecânica do jogo e assim projetá-los, fazendo com que perdessem o receio inicial em arriscar no desenvolvimento. Os alunos afirmaram que a oportunidade de criar um jogo do início, apesar do desafio, é extremamente motivador por ver o funcionamento do produto final.

Os alunos afirmaram ainda que durante a execução da oficina, a interação entre 
V Congresso Brasileiro de Informática na Educação (CBIE 2016)

Anais dos Workshops do V Congresso Brasileiro de Informática na Educação (CBIE 2016)

os colegas e professores, as demonstrações práticas e os desafios (minimaratona de programação) motivaram o aprendizado e a permanência nas aulas. Afirmaram ainda que conseguiram estimular o pensamento estratégico de forma descontraída, devido à ludicidade das aulas. Dos participantes, 93\% declararam que a metodologia despertou o interesse pelo desenvolvimento de jogos. Quanto às melhorias, foi sugerido que apresentassem novos motores de jogo que dessem mais suporte a elaboração da parte gráfica, principalmente de gráficos $3 \mathrm{D}$.

\section{Considerações Finais}

Este trabalho apresentou uma oficina de produção de jogos com o intuito de estimular aspectos multidisciplinares, intrínsecos ao processo de desenvolvimento de jogos, aplicados no ensino de programação para alunos do Ensino Médio. Planejamos a oficina a partir de lições aprendidas em execuções piloto. Avaliamos os projetos dos alunos sobre os aspectos de documentação, programação e jogabilidade.

Como resultados, percebemos que a fase de documentação auxiliou os alunos a planejarem previamente todo o jogo, evitando adicionar novas funcionalidades na etapa de implementação. No tocante a programação, encontramos elementos de lógica, abstração, iteratividade, representação de dados, controle de fluxo, sincronização e paralelismo nos projetos finais. Já no quesito jogabilidade, todos os projetos usaram as heurísticas de jogabilidade estimuladas.

A partir dos resultados alcançados, levantamos mais indícios para reforçar o argumento que o desenvolvimento de jogos digitais pode ter um impacto positivo quando adotado como fator motivacional no processo de ensino-aprendizagem. Ademais, acreditamos que o uso das heurísticas de jogabilidade possa ter influenciado na qualidade dos projetos desenvolvidos e na motivação dos alunos em desenvolver os jogos.

Foi observado que parte dos alunos, por mais que tenham se interessado pela programação, não tem vontade de seguir na área da TI. Entretanto, as habilidades trabalhadas nessa oficina podem complementar a formação dos alunos, independente da carreira que venham a seguir. A metodologia desenvolvida na oficina permitiu aos alunos estimular habilidades do Pensamento Computacional relacionadas à construção de algoritmos, abstração, simulação, paralelismo e automatização de soluções.

Como trabalhos futuros, no tocante à programação, planejamos inserir recomendações de boas práticas de organização e reutilização de código. Também pesquisaremos aspectos para avaliar a documentação produzida pelos alunos (storyboard e diagrama de elementos do jogo). Além disso, avaliaremos outros motores de jogos que permitam explorar mais funcionalidades para produção de jogos. Por último, investigaremos como habilidades do Pensamento Computacional podem ser estimuladas e avaliadas através de oficinas de desenvolvimento de jogos.

\section{Agradecimentos}

Os autores agradecem à CAPES pela concessão de bolsas do Programa Institucional de Bolsa de Iniciação à Docência - PIBID no período de realização deste trabalho. 
V Congresso Brasileiro de Informática na Educação (CBIE 2016)

Anais dos Workshops do V Congresso Brasileiro de Informática na Educação (CBIE 2016)

\section{Referências}

Andrade, M., Silva, C. \& Oliveira, T. (2013). Desenvolvendo games e aprendendo matemática utilizando o Scratch. In: Anais do XII Simpósio Brasileiro de Games e Entretenimento Digital (SBGames).

Barcelos, T. S. \& Silveira, I. F. (2012). Teaching computational thinking in initial series: An analysis of the confluence among mathematics and Computer Sciences in elementary education and its implications for higher education. In: XXXVIII Conferencia Latinoamericana En Informatica.

Barcelos, T. S., \& Silveira, I. F. (2013). Relações entre o Pensamento Computacional e a Matemática através da construção de Jogos Digitais. In: Anais do XII Simpósio Brasileiro de Games e Entretenimento Digital (SBGames).

Crenshaw, T. L., Chambers, E. W. \& Metcalf, H. (2008). A case study of retention practices at the University of Illinois at Urbana-Champaign. In: Special Interest Group on Computer Science Education (SIGCSE), v. 40, n. 1, p. 412-416.

França, R. S. \& Tedesco, P. (2015). Explorando o pensamento computacional no ensino médio: do design à avaliação de jogos digitais. In: Anais do XXIII Workshop sobre Educação em Computação (WEI).

Hernandez, C. C., Silva, L., Segura, R. A., Schimiguel, J., Ledón, M. F. P., Bezerra, L. N. M. \& Silveira, I. F (2010). Teaching programming principles through a game engine. In: CLEI Electronic Journal, v. 13, n. 2, p. 1-8.

Korhonen, H. \& Koivisto, E. (2006). Playability heuristics for mobile games. In: Proceedings of the 8th Conference on Human-computer Interaction with Mobile Devices and Services. ACM. p. 9-16.

Medeiros, T. J., Da Silva, T. R. \& Da Silva Aranha, E. H. (2013). Ensino de programação utilizando jogos digitais: uma revisão sistemática da literatura. In: Renote, v.11, n. 3.

Moratori, P.B. (2003). Por que utilizar jogos educativos no processo de ensino aprendizagem. Monografia. 33p. UFRJ, Rio de Janeiro.

Muratet, M., Torguet, P., Jessel, J. \& Viallet, F. (2009). Towards a serious game to help students learn computer programming. In: International Journal of Computer Games Technology, v. 2009, p. 3.

Rebouças, A. D. D. S., Marques, D. L., Costa, L. F. S., Silva, M. A. (2010). Aprendendo a ensinar programação combinando jogos e Python. In: Anais do Simpósio Brasileiro de Informática na Educação.

Scaico, P. D., Lima, A. A., Silva, J. B. B., Azevedo, S., Paiva, L. F., Raposo, E. H. S., Alencar, Y., Mendes, J. P. (2012). Programação no Ensino Médio: Uma Abordagem de Ensino Orientado ao Design com Scratch. In: Anais do Workshop de Informática na Escola.

Vegso, J. (2010). Interest in CS as a Major Drops Among Incoming Freshmen. In: Computing Research News, Vol.17, n.3. 